\title{
Three Different Cases of Breast Fibro adenomas
}

\section{*Sushila B Ladumor \\ Clinical Imaging Department, Weil Cornel Medical College, Qatar}

Submission: October 18, 2017; Published: November 14, 2017

"Correspondence Address: Sushila B Ladumor, Consultant Radiologist, Clinical Imaging Department, Hamad Medical Corporation, HGH, Doha, Qatar, Assistant Professor in Clinical Radiology, Weil Cornel Medical College, Doha, Qatar (WCMC-Q),

Email: drsbladumor@yahoo.com

Abstract

Fibro adenoma is a common benign breast lesion and results from excess proliferation of connective tissue. Fibro adenomas characteristically contain both stromal and epithelial cells. They usually occur in women between the ages of 10 and 40 years. It is the most common breast mass in the adolescent and young adult population [1]. Their peak incidence is between 25 and 40 years. Incidence decreases after 40 years. When found in an adolescent girl, the term juvenile fibro adenoma is more appropriate.

Keywords: Fibro adenoma; Benign; Malignant; Ultrasound (US); Doppler; Breast; US elastography

Case Report

Case 1

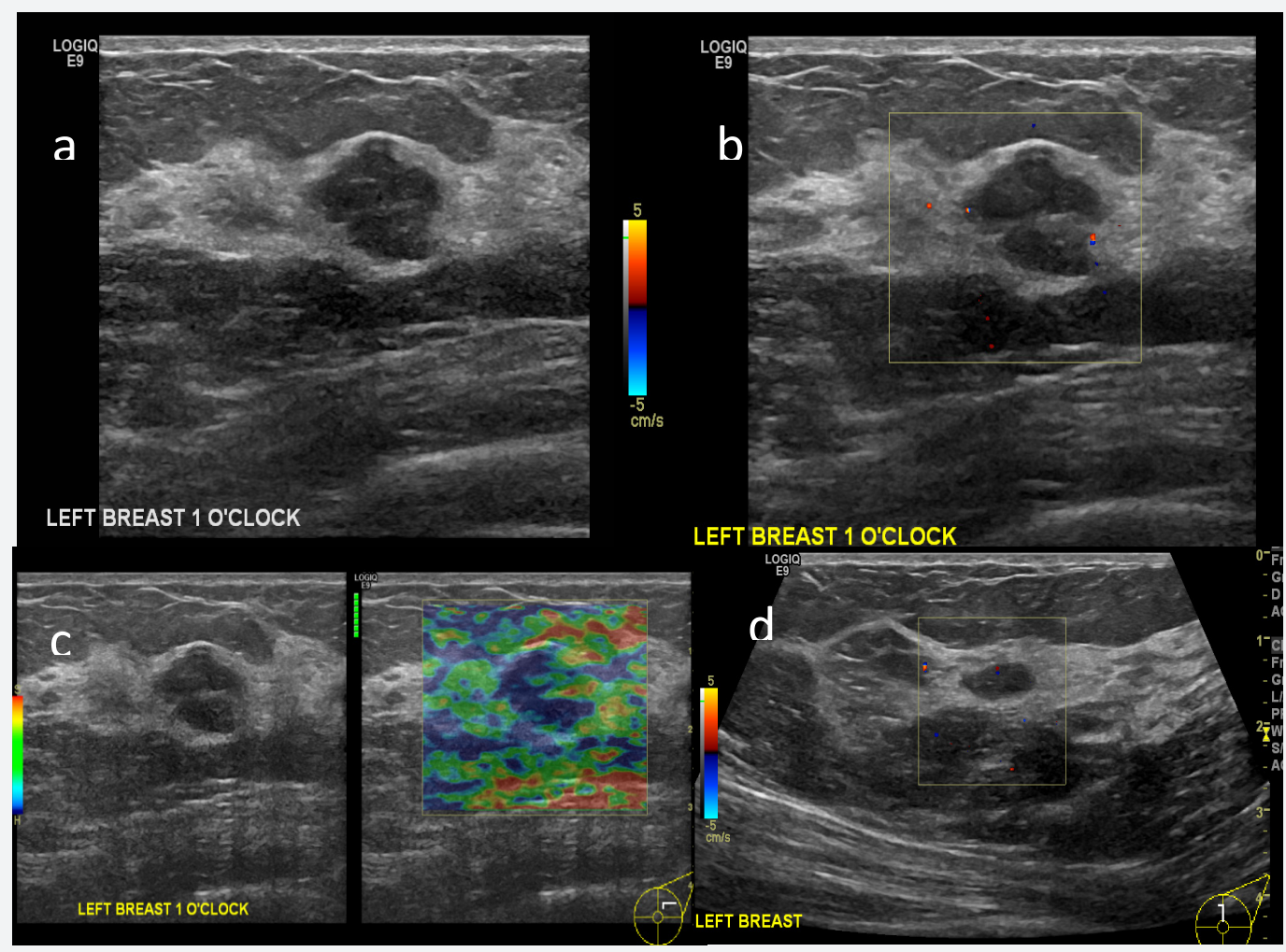

Figure 1: 26year old female patient with complaint of left breast mass. 


\section{Cancer Therapy \& Oncology International Journal}

US: Hypoechoic mass at 1'o"clock position in left breast with lobulated outline (Figure 1a) measures approximately $2.5 \times 2.0 \mathrm{~cm}$, showing no internal vascularity (Figure $1 \mathrm{~b}$ ), US elastography shows benign features as it shows more moreelastic areas and another small well defined hypoechoic nodule at 12 O'clock position with no color flow represents fibro adenoma (Figure 1).

\section{Impression-Case 1}

Lobulated hypo echoic nodule in left breast could represent fibro adenoma however needs tissue diagnosis for confirmation. BI-RADS 3-4a as no previous exam available. Another welldefined hypoechoic nodule in the left breast represents fibroadenoma.BI-RADS 2. Diagnosis of Left breast, core biopsy: Fibro epithelial lesion consistent with fibro adenoma.

\section{Case 2-Ultrasound}

Case 2

US: Right breast (Figures 2a \& 2b) ultrasound reveals lobulated hypoechoic lesion at 10 to 11 o'clock position at the site of palpable mass measures approximately 31 × $20 \mathrm{~mm}$ (b) demonstrated some peripheral vascularity (a). Left breast ultrasound (Figures 1c \& 1d) revealed hypo echoic lesion in sub areolar region at 9 o'clock position (c) at the site of palpable mass measures about $16 \times 14 \mathrm{~mm}$ with no internal vascularity in Doppler exam (not shown). Three hypoechoic nodules at 3 o'clock position measures about $24 \times 10 \mathrm{~mm}, 10 \times 3 \mathrm{~mm}$ and 7 x $4 \mathrm{~mm}$ (d)with no internal vascularity in Doppler exam (not shown) (Figure 2).

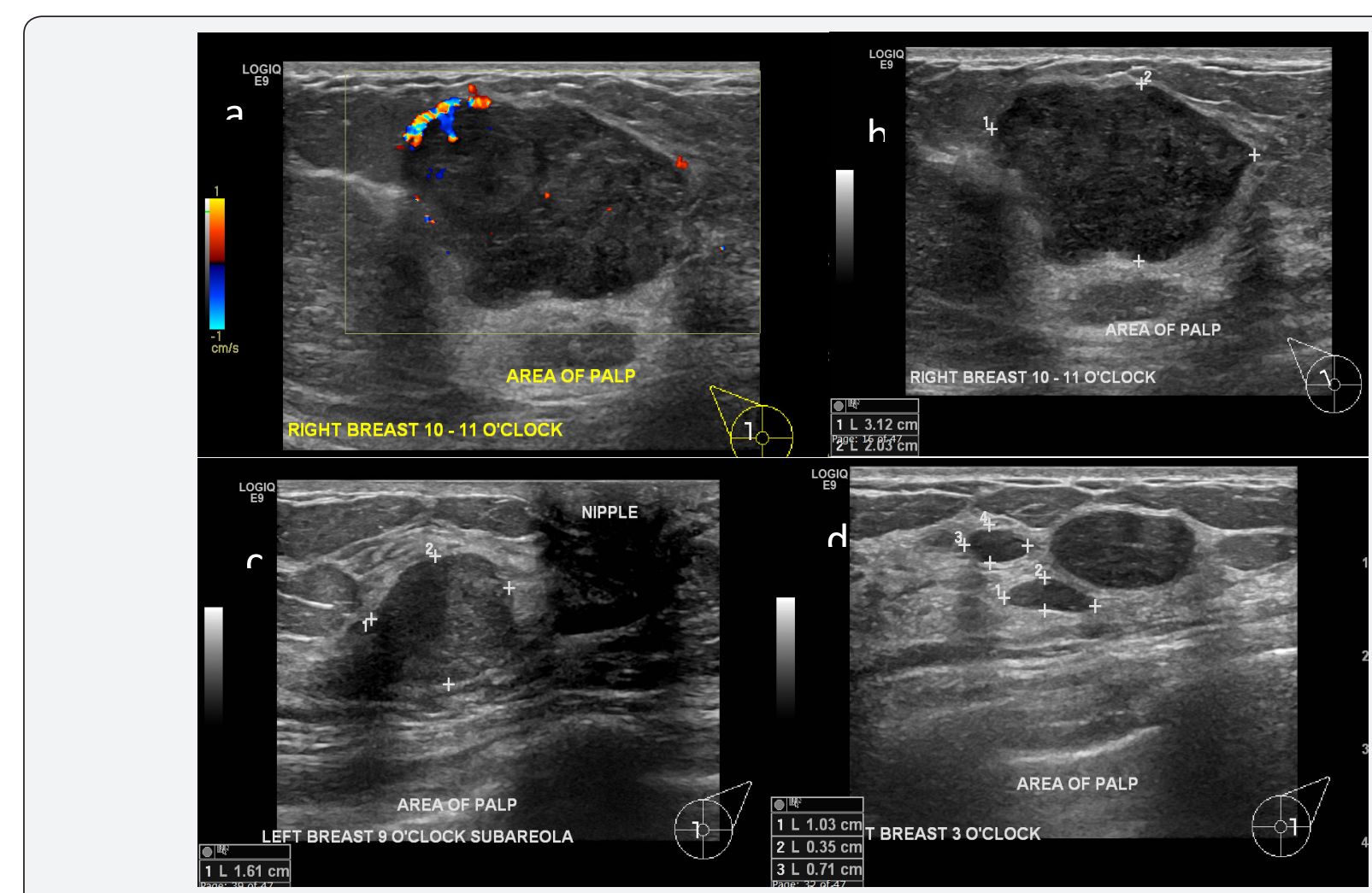

Figure 2: 30 year old female with complaint of bilateral breast masses.

\section{Impression-Case 2}

Above-described finding representing bilateral breast fibro adenomas. BI-RADS 3. For clinical correlation and short-term follow-up or tissue diagnosis from larger right breast mass.

i. Patient preferred tissue diagnosis;

ii. Diagnosis of Right breast, core biopsy;

iii. Fibro epithelial lesion consistent with fibro adenoma.

\section{Case 3}

US-Left breast US reveal well-defined hypo echoic mass at 2-3 O'clock position with smooth margin measures about 3.5 x $2.3 \mathrm{~cm}$ with speck of internal vascularity (Figure 1a). Right breast US (Figures $2 \mathrm{~b}, 3 \mathrm{c} \& 3 \mathrm{~d}$ ) reveals multiple small fibro adenoma. Fibro adenoma at 8-9 0'clock position appears iso to hypo-echoic (Figure 3).

\section{Impression: Case 3}

Above-described finding representing bilateral breast fibro adenomas. BI-RADS 3.

i. For clinical correlation and short-term follow-up or tissue diagnosis from larger left breast mass.

ii. Left breast mass removed surgically.

iii. Diagnosis: Fibro epithelial lesion consistent with fibro adenoma. 


\section{Cancer Therapy \& Oncology International Journal}

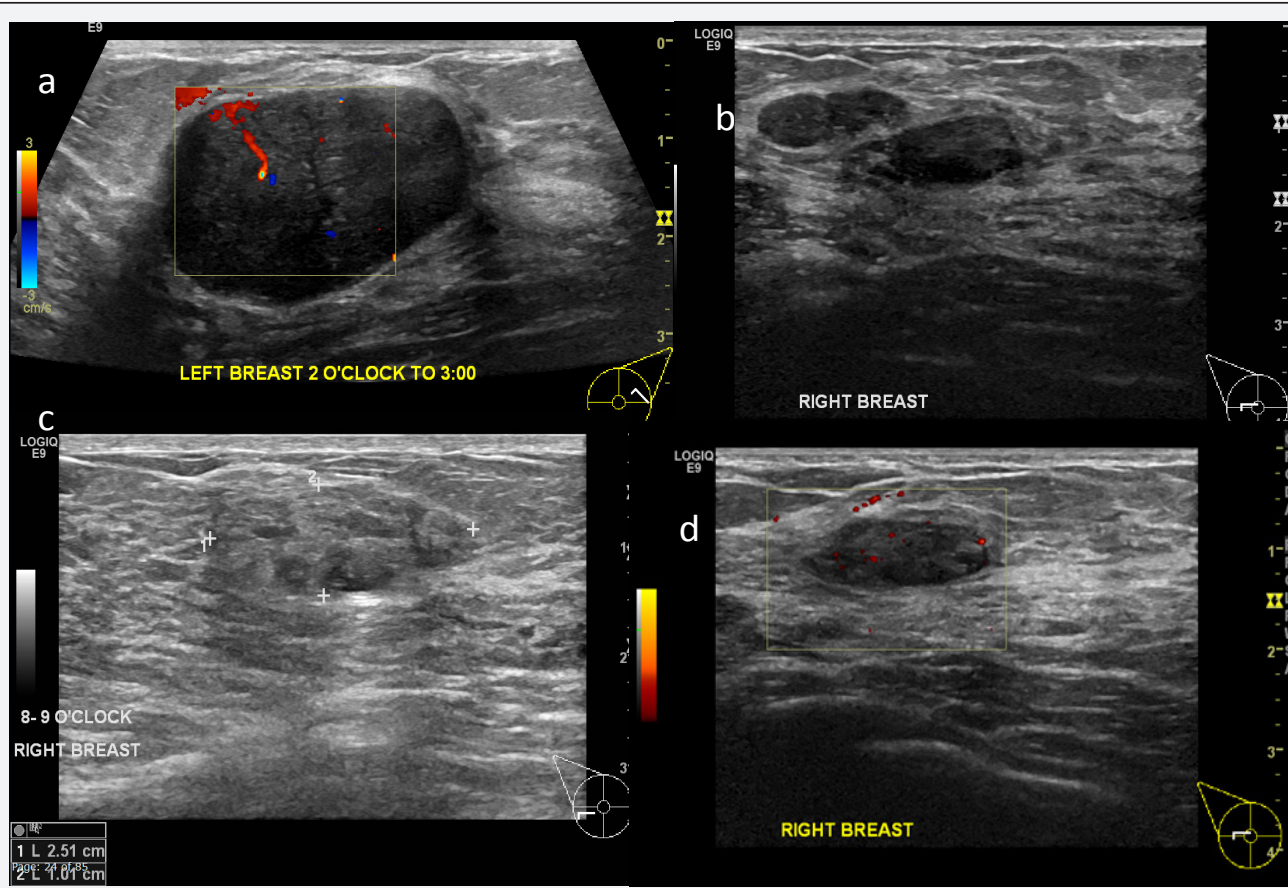

Figure 3: US: Left breast US reveal well-defined hypo echoic mass at 2-3 O'clock position with smooth margin measures about $3.5 \times 2.3 \mathrm{~cm}$ with speck of internal vascularity (image \# a). Right breast US (b, c \& d) reveals multiple small fibro adenoma. Fibro adenoma at 8-9 O'clock position appears iso to hypo-echoic (image \# c).

\section{Introduction}

Fibro adenomas are the most common solid breast masses excised in the adolescent population [1]. Classically, fibro adenomas are mobile non-tender breast lesions [1]. US scans show the typically benign features of benign solid lesions: a well-circumscribed hypo echoic mass that may be oval, round, or macro lobulated. Other features of benign solid masses at US include three or fewer circumscribed lobulations, wellcircumscribed margins, and horizontal orientation with transverse diameter greater than antero-posterior diameter. The diameter commonly ranges from 2 to $3 \mathrm{~cm}$ or less, although some may grow considerably larger. The term "giant fibro adenoma" is reserved for fibro adenomas that are $10 \mathrm{~cm}$ or more in diameter [1].

\section{Clinical Presentation}

The usual clinical presentation is in a woman of reproductive age with a mobile palpable breast lump. Due to their hormonal sensitivity, the fibro adenoma commonly enlarges during pregnancy and involute at menopause. Hence, they rarely present after the age of 40 years. The lesions are well defined and well circumscribed clinically and the overlying skin is normal. The lesions are not fixed to the surrounding parenchyma and slip around under the palpating hand, hence the colloquial term a breast "mouse".

\section{Discussion}

Fibro adenomas are benign fibro epithelial tumors that develop in the lobules at the ends of mammary gland ducts, which are the basic units of analysis at histopathologic assessment. Fibro adenomas are composed of epithelium and stroma, and they are the breast tumors most commonly found in adolescent girls and young women at clinical examination and histopathologic analysis. When palpable, fibro adenomas are smooth, mobile and firm or rubbery. In $15 \%$ of cases, multiple fibro adenomas are present. Fibro adenomas occasionally develop into very large masses, particularly in adolescent girls and young women; such masses are called juvenile giant fibro adenomas [2].

Radiologists must be familiar with a variety of benign breast conditions to confidently distinguish malignant disease from benign disease. Fibro adenomas are benign tumors composed of stromal and epithelial elements.These tumors are commonly seen in young women. Multiple or complex fibro adenomas may indicate a slightly increased risk for breast cancer; the relative risk of patients with such fibro adenomas is approximately twice that of patients of similar age without fibro adenomas.

The value of ultrasound in differencing cystic from a solid mass has been documented in numerous studies. Sonographic differentiation of benign from malignant solid breast tumors sometimes not possible with a good degree of accuracy, mainly because of their overlapping US features [3]. The fibro adenomas are usually hypo echoic and homogeneous in echo texture $(72 \%)$. Some of the fibro adenomas may have irregular margins. Intratumoral calcifications and rarely necrosis can be seen. State-of-the-art high-frequency transducers, geometric analysis of tumors, and real-time ultrasound-guided fine-needle aspiration biopsy should help to distinguish between benign and malignant solid breast masses in indeterminate cases. 


\section{Details about Ultrasound Imaging of the Breast}

Ultrasound is safe and painless, and produces pictures of the inside of the body using sound waves. Ultrasound imaging, also called ultrasound scanning or sonography, involves the use of a small transducer (probe) and ultrasound gel placed directly on the skin. High-frequency sound waves are transmitted from the probe through the gel into the body. The transducer collects the sounds that bounce back and a computer then uses those sound waves to create an image. Ultrasound examinations do not use ionizing radiation (as used in x-rays), thus there is no radiation exposure to the patient. Because ultrasound images are captured in real-time, they can show the structure and movement of the body's internal organs, as well as blood flowing through blood vessels.

Ultrasound imaging is a noninvasive medical test that helps physicians diagnose and treat medical conditions as well as very helpful for follow-up. Doppler ultrasound, also called color Doppler ultrasonography, is a special ultrasound technique that allows the physician to see and evaluate blood flow within the mass as well as in organs. Ultrasound imaging of the breast produces a picture of the internal structures of the breast. During a breast ultrasound examination the sonographer or physician performing the test may use Doppler techniques to evaluate blood flow or lack of flow in any breast mass. In some cases this may provide additional information as to the cause of the mass.

\section{Location of Fibro adenomas}

Although they can be located anywhere in the breast, there may be a predilection for the upper outer quadrant.

\section{Radiographic Features}

\section{Mammography}

Fibro adenomas have a spectrum of features from the well circumscribed discrete oval mass hypo- or isodense to the breast glandular tissue, to a mass with macrolobulations or partially obscured margin. Involuting fibro adenomas in older, typically postmenopausal patients may contain calcification, often producing the classic, coarse popcorn calcification appearance. In some cases the whole lesion is calcified. Calcification may also present as crushed stone-like micro calcification which makes differentiation from malignancy difficult.

\section{Breast Ultrasound}

Typically seen as a well-circumscribed, round to ovoid, or macro lobulated mass with generally uniform hypo echogenicity. Intralesional sonographically detectable calcification may be seen in $\sim 10 \%$ of cases [3] sometimes a thin echogenic rim (pseudo capsule) may be seen sonographically.

\section{Breast MRI}

T1: typically hypo intense or isointense compared with adjacent breast tissue

T2: can be hypo- or hyper intense
T1 C+ (Gd): can be variable but a majority will show slow initial contrast enhancement followed by a persistent delayed phase (type I enhancement curve); non-enhancing internal septations may be seen.

\section{Diagnosis}

These lesions are easily biopsied under ultrasound guidance. When a lesion has the typical features of a fibro adenoma on ultrasound and there are no clinical red flags they can be safely followed clinically. When lesions enlarge or have atypical imaging findings, ultrasound-guided core biopsy is a minimally invasive outpatient procedure that will give a diagnosis with virtually no complications [4].

Depending on where you work, there may be a maximum diameter above which a biopsy should be done if no previous imaging is available. There is significant local variation in this regard. The reason for intervention based on size is that a phylloides tumor may be indistinguishable from a fibro adenoma on ultrasound. A maximum diameter of $2.5 \mathrm{~cm}$ may be a useful benchmark for biopsy if you have no previous imaging. Interval enlargement is an indication for biopsy [5].

\section{Pathology}

A fibro adenoma is a type of adenomatous breast lesion. It contains epithelium and has minimal malignant potential. Multiple fibro adenomas occur in $10-15 \%$ of patients. Patients with multiple fibro adenomas tend to have a strong family history of these tumors.

\section{Treatment and prognosis}

They are benign lesions with minimal or no malignant potential. The risk of malignant transformation is extremely low and has been of reported to range around be $0.0125-0.3 \%$.

Indications for biopsy include:

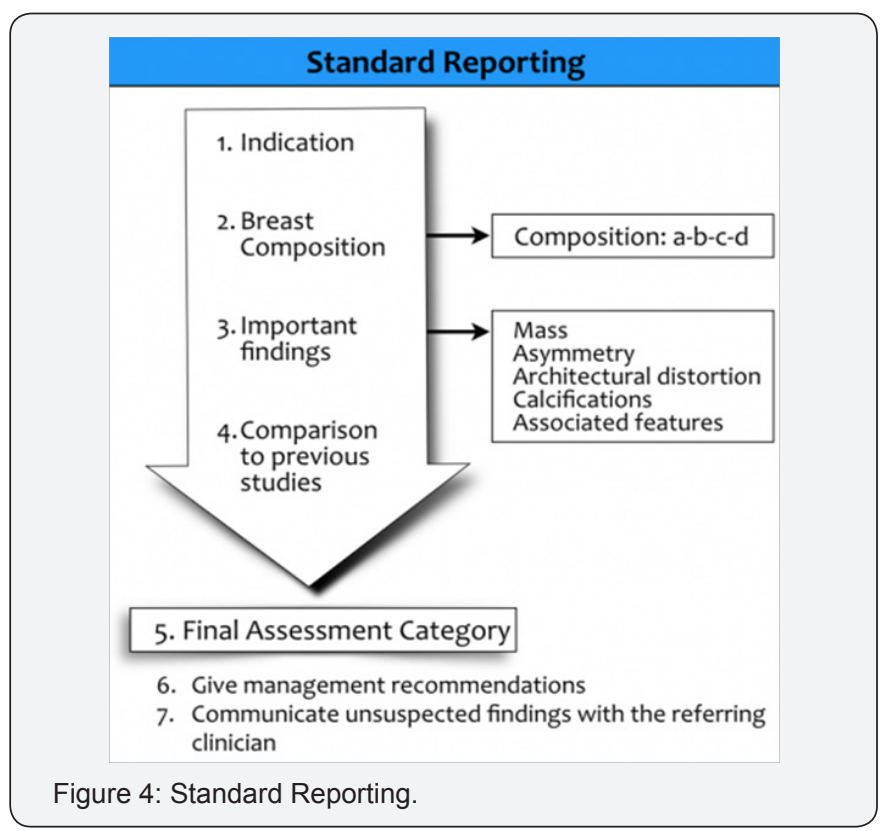




\section{Cancer Therapy \& Oncology International Journal}

i. Enlarging lesion

ii. Atypical findings on ultrasound

iii. A lesion above $2.5-3.0 \mathrm{~cm}$ where there are no previous studies for comparison

Table 1: Ultrasound Lexicon.

\begin{tabular}{|c|c|c|}
\hline \multicolumn{3}{|c|}{ Ultrasound Lexicon } \\
\hline \multirow{3}{*}{ Breast Composition } & a. Homogeneous-Fat & \\
\hline & b. Homogeneous-Fibro glandular & \\
\hline & c. Heterogeneous & \\
\hline \multirow[t]{5}{*}{ Mass } & Shape & Oval-round-irregular \\
\hline & Margin & $\begin{array}{l}\text { Circumscribed or Not-Circumscribed: } \\
\text { indistinct, angular, microlobulated, spiculated }\end{array}$ \\
\hline & Orientation & Parallel-not parallel \\
\hline & Echo pattern & $\begin{array}{l}\text { Anechoic-hyperechoic-complex cystic/solid } \\
\text { hypoechoic-isoechoic-heterogeneous }\end{array}$ \\
\hline & Posterior & No features-enhancement-Shadowing \\
\hline Associated features & \multicolumn{2}{|c|}{ In mass-Outside mass-Intraductal } \\
\hline Associated features & \multicolumn{2}{|c|}{$\begin{array}{l}\text { Architectural distortion-duct changes-skin thickening-skin retraction-edema-vascularity(absent, } \\
\text { internal,rim)-elasticity }\end{array}$} \\
\hline Special cases (Cases with a unique diagnosis) & \multicolumn{2}{|c|}{$\begin{array}{l}\text { Simple cyst-clustered microcysts-complicated cyst-mass in or skin- foreign body(including } \\
\text { implants)-intramammary lymph node-AVM-Monodor disease-postsurgical fluid collection-fat } \\
\text { necrosis }\end{array}$} \\
\hline
\end{tabular}

Table 2: Final Assessment Categories.

\begin{tabular}{|c|c|c|c|}
\hline \multicolumn{4}{|c|}{ Final Assessment Categories } \\
\hline \multicolumn{2}{|c|}{ Category } & Management & Likelihood of cancer \\
\hline 0 & $\begin{array}{c}\text { Need Additional Imaging or prior } \\
\text { examinations }\end{array}$ & $\begin{array}{l}\text { Recall for additional imaging and/ } \\
\text { or await prior examinations }\end{array}$ & $\mathrm{n} / \mathrm{a}$ \\
\hline 1 & Negative & Routine screening & Essentially $0 \%$ \\
\hline 2 & Benign & Routine screening & Essentially $0 \%$ \\
\hline 3 & Probably Benign & $\begin{array}{l}\text { Short interval-follow-up( } 6 \text { month) } \\
\text { or continued }\end{array}$ & $>0 \%$ but $<2 \%$ \\
\hline 4 & Suspicious & Tissue diagnosis & $\begin{array}{l}\text { 4a. low suspicion for malignancy } \\
\qquad>2 \% \text { to }<10 \% \\
\text { 4b. moderate suspicion for } \\
\text { malignancy }(>10 \% \text { to }<50 \% \\
\text { 4c. high suspicion for malignancy } \\
\quad(>50 \% \text { to }<95 \%)\end{array}$ \\
\hline 5 & Highly suggestive of malignancy & Tissue diagnosis & $>95 \%$ \\
\hline 6 & Known biopsy-proven & $\begin{array}{l}\text { Surgical excision when clinical } \\
\text { appropriate }\end{array}$ & $\mathrm{n} / \mathrm{a}$ \\
\hline
\end{tabular}

\section{Conclusion}

In the adolescent population benign breast findings are overwhelmingly the most common cause of symptomatic breast abnormalities. A careful physical examination with clinical follow-up and US help for the evaluation of patient symptoms. Reassurance should be provided for both the patients and parents in cases of benign findings at both clinical examination and US. When US scans are negative, however, the level of clinical concern should guide management. Given the overwhelmingly benign causes, surgery should be the last resort and reserved for only lesions that enlarge or have highly suspect features. iv. Patient peace of mind: some patients are simply not happy with a palpable mass in the breast without a histological diagnosis; this is a valid and reasonable indication for biopsy (Tables $1 \&$ 2) (Figure 4). 
5. Amin AL, Purdy AC, Mattingly JD, Kong AL, Termuhlen PM (2013) Benign breast disease. Surg Clin North Am 93(2): 299-308.

This work is licensed under Creative Commons Attribution 4.0 License DOI:_10.19080/CTOIJ.2017.08.555726 